\title{
Unusual Cases of Multiple Symmetrical Lipomatosis with Neurological Disorders
}

\author{
Nikolaos I. Triantafyllou, MD; loannis Zalonis, PhD; Grigoris Kararizos, MD; \\ Konstantinos Gkiatas, MD; Fotini Christidi, BA and Evangelia Kararizou, MD
}

\begin{abstract}
Multiple symmetrical lipomatosis (MSL) is a rare disorder of unknown etiology defined as the presence of multiple and symmetrical fatty accumulations, usually involving the upper trunk, neck and head. Frequently associated findings include diabetes mellitus, hyperlipidemia, liver disease, hypothyroidism and polyneuropathy of unknown origin, but nevertheless, there are published reports of cognitive disorders in patients with MSL. We describe two unusual cases (38-year-old and 45-year-old Greek men) of MSL who presented with polyneuropathy and memory disorders. This is the first description of memory disorders in patients with MSL. We propose that MiniMental State Examination and assessment of cognitive functions should be performed for all patients with MSL.The underlying mechanism in our patients remains unknown, and this question should be the subject of a future study.
\end{abstract}

Keywords: Madelung's disease; Memory disorders; Multiple symmetrical lipomatosis (MSL); Polyneuropathy

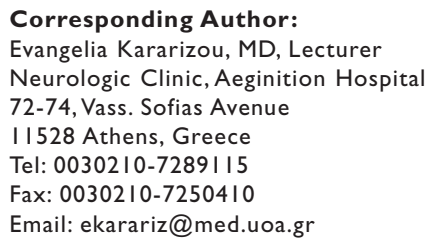

Received: December 3I, 2008

Revised: February 23, 2009

Accepted: March 18, 2009

doi: $10.3121 / \mathrm{cmr} .2009 .838$
$\mathrm{M}$ Benjamin Brodie in 1846, ${ }^{1}$ but Otto Madelung reported the first series of patients in $1888 . .^{2}$ It is a rare disorder of unknown etiology defined as the presence of multiple and symmetrical fatty accumulations, usually involving the upper trunk, neck and head. ${ }^{3}$ Several descriptive names have been associated with it, including benign symmetrical lipomatosis, MSL, and symmetrical adenolipomatosis. ${ }^{4}$ The disorder usually occurs in middle-aged male alcoholics.

Frequently associated findings include diabetes mellitus, hyperlipidemia, liver disease, hypothyroidism, and polyneuropathy of unknown origin, but nevertheless, there are published reports of cognitive disorders in patients with MSL.5,6 We describe two unusual cases of MSL associated with memory disorders. This is the first description of memory disorders in patients with MSL.

\section{Case Presentations}

Case 1

A 38-year-old man, who is a heavy alcohol drinker, developed a lipomatous mass in the right postauricular area which slowly enlarged. Similar masses started subsequently developing in a symmetrical manner over the entire body. The patient was admitted to the Department of Oral and Maxillofacial Surgery at the General Hospital of Athens. The physical examination showed an enlargement of the postauricular, retroauricular, occipital and upper back areas. A faciocervicothoracic computed tomography scan (CT) showed a fatty accumulation in the referred areas. Magnetic resonance imaging revealed large masses of unencapsulated adipose tissue. Histological study of the samples obtained by 

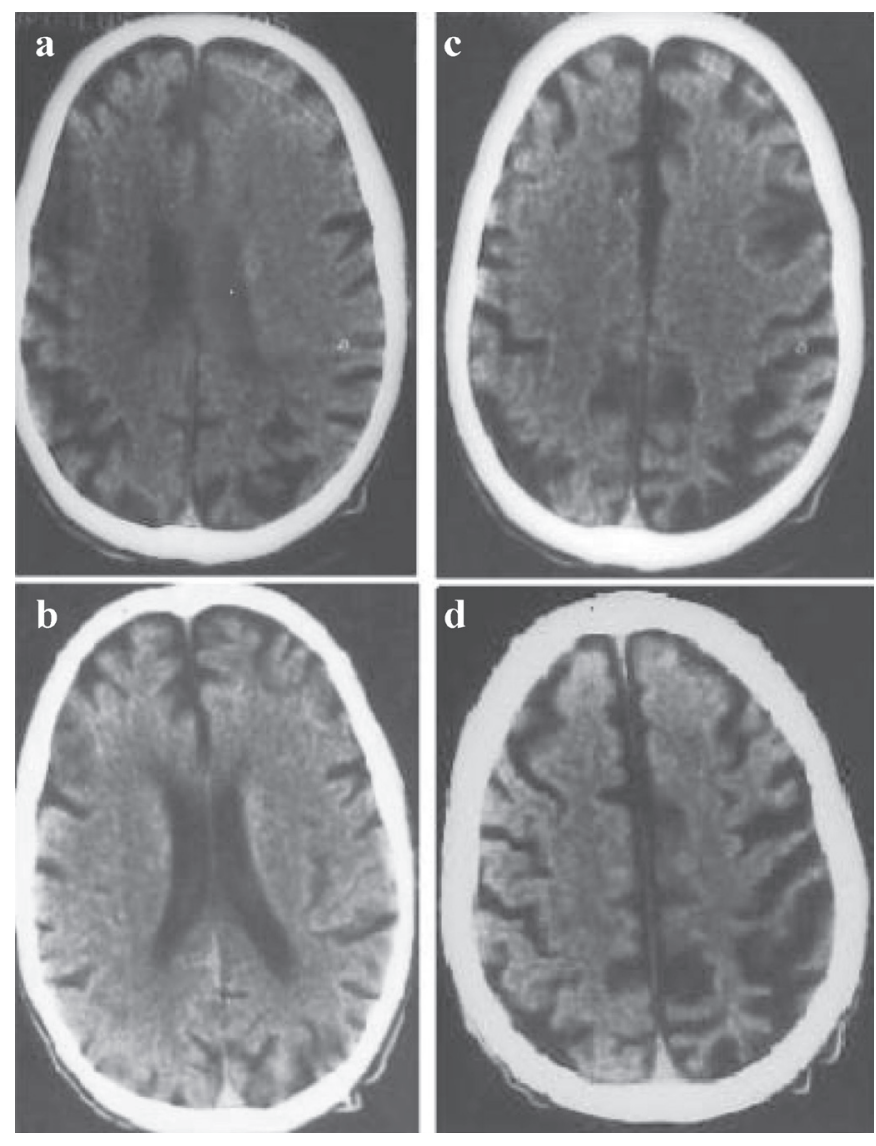

Figure 1. CT scan showed brain atrophy. Case 1 before treatment (a) and after treatment (b). Case 2 before treatment (c) and after treatment (d).

needle-aspiration of the lesions showed hypervascularized fibroadipose tissue, and MSL was diagnosed. In recent years he has undergone several surgical excisions of the masses, and lipomatous evolution stopped developing in 2004. Four months before the appearance of the last lipomatous mass he developed numbness of the toes that gradually extended to the knee. At this time he was seen at our clinic with the complaint of painful weakness and burning feet. Neurological examination revealed sensorimotor polyneuropathy. Nerve conduction studies showed reduction of the sensory and motor conduction velocities. Routine hematological, complete biochemical and serological tests have been obtained which were negative. Endocrine test results were normal. The patient was seen again in follow-up examination 1-year later. At this time he complained of dysaesthesias in all four extremities and recent memory disturbances. A brain scan revealed signs of moderate brain atrophy. The Mini-Mental State Examination (MMSE) was nearly normal with a score of 26, but in follow-up examinations, 2- and 3-years later, his score decreased to 24 and 21, respectively. On the last CT brain scan there were also signs of progressive atrophy (figure $1 \mathrm{a}$ and $1 \mathrm{~b}$ ).

At the time of the 26 and 24 MMSE scores, comprehensive neuropsychological assessment was performed (table 1).
According to test-retest findings, our patient presented cognitive deterioration, with defective control of complex attention and progressive neuropsychological deficits in memory function (immediate memory span, episodic/prose memory, efficiency of learning strategy, visual recall), visuomotor and spatioperceptual dexterities, as well as visual reasoning and executive processes (verbal fluency, cognitive flexibility, establishing, maintaining, and shifting cognitive sets as a response to changing environmental demands). Contrary to fluid intelligence, crystallized verbal intelligence remains within the normal performance range.

Case 2

A 45-year-old man reported multiple, large neck masses that had enlarged progressively during the last 5 years. He was a mild to moderate drinker of alcohol. CT confirmed the presence of a large lipoma infiltrating the muscular planes. The patient was diagnosed as having MSL based on the physical examination findings and subcutaneous fat tissue biopsy. A fatty mass was excised. Histologic study confirmed the initial diagnosis. The patient presented to our hospital complaining of numbness of the toes, anxiety, insomnia and memory disorders. Neurological examination revealed sensory neuropathy. Electrophysiological study confirmed the diagnosis. There were not any clinical or laboratory indicators of other disease. Complete biochemical analysis was normal. A brain CT scan showed atrophy (figure 1c and 1d).

At the time of hospitalization, MMSE was 25, decreasing progressively to 23 and 20 in follow-up examinations. Neuropsychological examinations at the time of the 25 and 23 MMSE scores (time interval of 2 years) revealed the patient's diffuse non-age-related cognitive deterioration in multiple cognitive domains, with prominent memory deficits (working memory, episodic/prose memory, learning ability, efficiency of learning strategies, visual memory) and significant deficits on complex attention, visuomotor and spatioperceptual abilities, concept formation-reasoning, and executive functions (table 1). It was only his performance on measures of common sense judgment/practical reasoning, vocabulary and general knowledge that remained within the normal performance range. Fluid and crystallized intelligence were significantly affected.

\section{Discussion}

MSL is characterized by diffuse deposits of fat arranged symmetrically around the neck and shoulder girdle. It is seen more frequently in middle-age men of Mediterranean descent who have a history of heavy alcohol consumption. The masses are nonencapsulated and can eventually reach a very large size. The diagnosis is usually made by physical examination and clinical history, but nuclear magnetic resonance imaging, CT, and biopsy may be helpful. The etiology remains unclear. MSL has been classified as type I (for some authors the real Madelung's disease) characterized by masses distributed in the upper half of the body, weight loss and possible deep accumulation of adipose tissue 
Table 1. Neuropsychological test-retest findings.

\begin{tabular}{|c|c|c|c|c|c|}
\hline \multirow{3}{*}{ Neuropsychological tests } & \multicolumn{4}{|c|}{ Score } & \multirow{3}{*}{ Normal range / M (SD) } \\
\hline & \multicolumn{2}{|c|}{$1^{\text {st }}$ patient } & \multicolumn{2}{|c|}{$2^{\text {nd }}$ patient } & \\
\hline & $1^{\text {st }}$ exam & $2^{\text {nd }}$ exam & 1st exam & $2^{\text {nd }}$ exam & \\
\hline TMT-A & 91 & 129 & 87 & 156 & $44.90(13.82)$ \\
\hline TMT-B & 186 & 253 & 423 & 500 & 100.96 (33.92) \\
\hline 3W3S & 4 & 3 & 3 & 1 & $4-6$ \\
\hline BSRT-Im.recall & 11 & 9 & 6 & 6 & $12-21$ \\
\hline BSRT-Del.recall & 10 & 7 & 1 & 0 & $12-21$ \\
\hline RCFT-copy & 22 & 18 & 13 & 14 & $32-36$ \\
\hline RCFT-Im.recall & 14 & 10 & 2 & 0 & $18-36$ \\
\hline RAVLT 1st repetition & 5 & 4 & 3 & 3 & $6.2(1.4)$ \\
\hline RAVLT 1 st_-5th repetition $^{\text {th }}$ & 44 & 38 & 23 & 19 & $47.4(8.8)$ \\
\hline RAVLT retention & 7 & 6 & 4 & 3 & $9.6(3.0)$ \\
\hline COWAT & 26 & 22 & 25 & 20 & $27-62$ \\
\hline WCST-C & 4 & 3 & 3 & 3 & $>4$ \\
\hline WCST-PR.E & 27 & 35 & 38 & 41 & $<24$ \\
\hline WCST-CLR & 44 & 36 & 32 & 26 & $>48$ \\
\hline WAIS information & 9 & 9 & 9 & 9 & $10(1.5)$ \\
\hline WAIS comprehension & 9 & 10 & 10 & 9 & $10(1.5)$ \\
\hline WAIS arithmetic & 7 & 9 & 6 & 5 & $10(1.5)$ \\
\hline WAIS similarities & 7 & 7 & 5 & 2 & $10(1.5)$ \\
\hline WAIS digit span & 9 & 8 & 3 & 3 & $10(1.5)$ \\
\hline WAIS vocabulary & 9 & 9 & 8 & 8 & $10(1.5)$ \\
\hline WAIS digit symbol & 2 & 0 & 6 & 3 & $10(1.5)$ \\
\hline WAIS picture completion & 2 & 0 & 3 & 1 & $10(1.5)$ \\
\hline WAIS block design & 4 & 0 & 6 & 4 & $10(1.5)$ \\
\hline WAIS picture arrangement & 6 & 0 & 3 & 3 & $10(1.5)$ \\
\hline WAIS object assembly & 2 & 0 & 3 & 3 & $10(1.5)$ \\
\hline Verbal IQ & 90 & 91 & 80 & 78 & $100(15)$ \\
\hline Performance IQ & 62 & 61 & 66 & 65 & $100(15)$ \\
\hline Full IQ & 77 & 73 & 73 & 71 & $100(15)$ \\
\hline
\end{tabular}

TMT, Trail Making Test-part A and part B; 3W3S, 3 Words 3 Shapes Test; BSRT, Babcock Story Recall Test-Immediate and Delayed Recall; RCFT, Rey's Complex Figure Test-Copy and Immediate Recall; RAVLT, Rey Auditory Verbal Learning Test $1^{\text {st }}$ repetition, $1^{\text {st }} 5^{\text {th }}$ repetitions, retention (delayed recall); COWAT, Controlled Oral Word Association Test; WCST, Wisconsin Card Sorting Test categories completed, preservative errors, conceptual level of responses; WAIS, Wechsler Adult Intelligence Scale.

(ie, mediastinal), and type II which is more difficult to differentiate from simple obesity with diffuse involvement, weight gain and the absence of deep involvement. . $^{3,4}$

The two cases reported here can be classified in the type I group. They are associated with the same neurological manifestations, polyneuropathy and memory disorders. The polyneuropathy affects the cases with MSL as mentioned by other authors. ${ }^{4-8}$ It may appear as an autonomic, sensory or motor neuropathy with proximal predominance, and may be associated with neuropathic skin lesions and Charcot's arthropathy. ${ }^{9}$ Neuropathy is a frequent complication of alcoholism but many publications distinguish polyneuropathy in MSL patients from alcoholism. ${ }^{10}$

We could not find other reports of memory disorders in patients with MSL. Both patients presented with memory disorders, decreased MMSE and cerebral atrophy on the CT brain scan. Neuropsychological tests revealed significant neuropsychological deficits.

Cognitive impairment is frequently observed in patients with alcohol misuse or alcohol addiction. ${ }^{11}$ Multiple cognitive functions are reduced in these patients, but frontal lobe functions, such as planning and abstract thinking, are most frequently affected. Alcohol amnestic syndrome, alcohol dementia, and the Wernicke-Korsakoff syndrome constitute distinct entities. It is well known that chronic alcoholism results in brain damage and dysfunction. Alcohol and its metabolite acetaldehyde are directly neurotoxic. Alcoholics are thiamine deficient as a result of poor diet, gastrointestinal disorders and liver disease. In addition, both alcohol and acetaldehyde have direct toxic effects on thiamine-related enzymes in the liver and brain. ${ }^{12}$ MSL has been related to high levels of alcohol consumption, but the etiopathogenetic role of alcohol is not yet known. In the study of Klopstock 
et al, ${ }^{13}$ abnormal mitochondrial status has been observed in MSL patients. Yet, Heckmann et $\mathrm{al}^{14}$ have reported a case of a female, heavy drinker, MSL patient with mild frontal atrophy, without neuropsychological impairment or mitochondrial abnormalities. In our study, the first patient was a heavy drinker, but the use of alcohol in the second was mild to moderate. According to criteria based on the Diagnostic and Statistical Manual of Mental Disorders, 4th edition, our second patient did not complete the criteria for alcoholrelated dementia, while the first patient fulfills the criteria for possible alcohol-related dementia. ${ }^{15}$

A point of interest is the possible relationship between the cognitive impairment and alcoholism, which is almost constantly present, rather than a possible relationship between the cognitive dysfunction and the MSL. It is well documented that excessive drinking is related to a higher risk of dementia, and thus, we cannot rule out the possibility of alcohol-related cognitive impairment in our patients. Despite the fact that many of the patients with MSL are heavy drinkers and many incur dementia, we could not find other publications with this disorder. Another point is the young age of our patients and the normal liver and complete serological examination in both of them.

In conclusion, these are two patients with diagnosed MSL, polyneuropathy and memory disorders. Although alcohol is associated with dementia, there were no other publications with MSL. The underlying mechanism in our patients remains unknown and this question should be the subject of a future study.

\section{References}

1. Brodie BC. Clinical lectures on surgery delivered at St. George's Hospital. Philadelphia: Lea \& Blanchard; 1846. 201-210.

2. Madelung OW. Ueber den Fetthals. Arch Klin Chir 1888; 37:106-130.

3. Hirose A, Okada Y, Morita E, Tanaka Y. Benign symmetric lipomatosis associated with alcoholism. Intern Med 2006;45:1001-1005.

4. Enzi G, Biondetti PR, Fiore D, Mazzoleni F. Computed tomography of deep fat masses in multiple symmetrical lipomatosis. Radiology 1982;144:121-124.

5. Saiz Hervás E, Martín Llorens M, López Alvarez J. Peripheral neuropathy as the first manifestation of Madelung's disease. Br J Dermatol 2000;143:684-686.

6. Teplitsky V, Huminer D, Dux S, Learman Y, Zoldan J, Pitlik SD. Multiple symmetric lipomatosis presenting with polyneuropathy. Isr J Med Sci 1995;31:693-695.

7. Enzi G, Angelini C, Negrin P, Armani M, Pierobon S, Fedele D. Sensory, motor, and autonomic neuropathy in patients with multiple symmetric lipomatosis. Medicine (Baltimore) 1985;64:388-393.

8. Pollock M, Nicholson GI, Nukada H, Cameron S, Frankish P. Neuropathy in multiple symmetric lipomatosis: Madelung's disease. Brain 1988;111:1157-1171.

9. Biasi D, Caramaschi P, Carletto A, Baracchino F, Botto M, Pacor ML, Bambara LM. [Symmetric multiple lipomatosis with Charcot's joint and neuropathic ulcer. description of a clinical case]. Minerva Med 1993;84:135-139.
10. Naumann M, Schalke B, Klopstock, Reichmann H, Lange KW, Wiesbeck G, Toyka KV, Reiners K. Neurological multisystem manifestation in multiple symmetric lipomatosis: a clinical and electrophysiological study. Muscle Nerve 1995; 18:693-698.

11. Solfrizzi V, D'Introno A, Colacicco AM, Capurso C, Del Parigi A, Baldassarre G, Scapicchio P, Scafato E, Amodio M, Capurso A, Panza F; Italian Longitudinal Study on Aging Working Group. Alcohol consumption, mild cognitive impairment, and progression to dementia. Neurology 2007;68:1790-1799.

12. Butterworth RF. Pathophysiology of alcoholic brain damage: synergistic effects of ethanol, thiamine deficiency and alcoholic liver disease. Metab Brain Dis 1995;10:1-8.

13. Klopstock T, Naumann M, Schalke B, Bischof F, Seibel P, Kottlors M, Eckert P, Reiners K, Toyka KV, Reichmann H. Multiple symmetric lipomatosis: abnormalities in complex IV and multiple deletions in mitochondrial DNA. Neurology 1994;44:862-866

14. Heckmann JG, Kraus B, Stefan H, Neundörfer B. Progressive muscle pain, weight gain, fatigue and unusual body shape change. Eur J Neurol 2000;7:243-244.

15. Oslin D, Atkinson RM, Smith DM, Hendrie H. Alcohol related dementia: proposed clinical criteria. Int J Geriatr Psychiatry 1998;13:203-212.

\section{Author Affiliations}

Nikolaos I. Triantafyllou, MD*; Ioannis Zalonis, PhD*; Fotini Christidi, BA*; Evangelia Kararizou, MD*; Grigoris Kararizos, $M D^{\dagger}$; Konstantinos Gkiatas, $M D^{\dagger}$

*Department of Neurology, Athens National University, Eginition Hospital, Athens, Greece; and 'Air Force Hospital, Neurological Clinic, Greece 